\title{
A novel reduced graphene oxide decorated with halloysite nanotubes (HNTs- $d$-rGO) hybrid composite and its flame-retardant application for polyamide 6
}

\author{
L. L. Li, S. H. Chen, W. J. Ma, Y. H. Cheng, Y. P. Tao, T. Z. Wu, W. P. Chen, Z. Zhou, M. F. Zhu*
}

State Key Laboratory for Modification of Chemical Fiber and Polymer Materials, College of Materials Science and Engineering, Donghua University, Shanghai 201620, People's Republic of China

Received 10 December 2013; accepted in revised form 24 February 2014

\begin{abstract}
The improvement of flame-retardant properties of polyamide 6 (PA6) was achieved by using reduced graphene oxide decorated with halloysite nanotubes (HNTs- $d$-rGO) hybrid composite as the additive in PA6 matrix. The intimate integration of reduced graphene oxide (rGO) and halloysite nanotubes (HNTs) through a three-step chemical functionalization, enabled the combination of their unique physical and chemical characteristics together. The nanostructure of HNTs- $d$ rGO was determined by Fourier transform infrared spectroscopy (FTIR), X-ray photoelectron spectroscopy (XPS) and transmission electron microscopy (TEM). A morphological study revealed that HNTs- $d$-rGO was dispersed uniformly in PA6 matrix. From the results of cone calorimetry measurements, the fire retardant properties of PA6 were further improved with the addition of HNTs- $d$-rGO when compared with that of either HNTs, or GO, or a mixture of HNTs and GO (HNTs$m$-GO) used in PA6 matrix. The results indicate clearly that higher flame-retardant activity of the integrated HNTs- $d$-rGO nanostructures than that of the simple mixture verifies the importance of the intimate integration between HNTs and rGO, which ascribe to the combination of the stable silica layer created by HNT and the barrier effect of rGO.
\end{abstract}

Keywords: nanocomposites, graphene oxide, halloysite nanotubes (HNTs), polyamide 6

\section{Introduction}

Polyamide 6 (PA6), a commercially important semicrystalline polymer, is widely used in engineering plastics, film and fibers [1-4], which usually causes fires and consequently leads to huge loss of human lives and property, which, to a certain degree, limits the range of applications. We demonstrate a novel strategy to improve the flame-retardant properties of PA6 by using reduced graphene oxide decorated with halloysite nanotubes (HNTs- $d$-rGO) hybrid composite.

Graphene, exhibiting outstanding electronic, mechanical [5-8] and thermal $[9,10]$ properties, is emerging as a rising star in the field of flame-retardant applications due to its endothermic and stable struc- ture [10-13]. For example, the peak heat release rate (PHRR) of polyvinyl alcohol (PVA) filled with graphene is reduced by $49 \%$ [11]. However, in order to achieve fire resistance, the content of graphene used as flame retardant in polymer should be relatively high. Besides, nanocomposites consisting of polymer and clay, such as montmorillonite and kaolinite, are widely used to improve the flame-retardant properties of polymers $[14,15]$. Halloysite nanotubes (HNTs), one kind of nanoclay, have attracted research attention as fillers for polymer nanocomposites due to their improvement of mechanical [16, 17], thermal [18], crystallization [19, 20] properties of polymer. Moreover, recent studies showed that HNTs enable to enhance the fire-retar-

\footnotetext{
*Corresponding author, e-mail: zhumf@dhu.edu.cn

(C) BME-PT
} 
dant performance of polymer composite, which is attributed to the formation of thermal insulation barrier during fire [21]. Therefore, the combination of reduced graphene oxide (rGO) and HNTs as additive may be considered as an efficient method to get good fire retardant properties in polymer.

Herein, we demonstrate a novel strategy to functionalize and decorate rGO with HNTs through a three-step chemical reaction. Finally, rGO decorated with HNTs (HNTs- $d$-rGO) was prepared and incorporated into PA6 for fire retardant applications.

\section{Experimental}

\subsection{Materials}

Graphite F-000 $(\leq 2 \mu \mathrm{m})$ was purchased from Shanghai Yifan graphite Co., Ltd. (Shanghai, China). Polyamide 6 shows a melt flow rate (MFR) of $23.89 \mathrm{~g} / 10 \mathrm{~min}$ provided by Jiangsu Haiyang Chemical Fiber Co., Ltd. (China). Halloysite nanotubes (HNTs) was supplied by Jinyangguang Ceramics Co. Ltd. (Zhengzhou, China). Sulphuric acid $\left(\mathrm{H}_{2} \mathrm{SO}_{4}\right.$, 98\%), hydrogen peroxide solution $\left(\mathrm{H}_{2} \mathrm{O}_{2}, 30 \%\right)$, hydrochloric acid $(\mathrm{HCl}, 37 \%)$, potassium permanganate $\left(\mathrm{KMnO}_{4}\right)$, dimethylformamide (DMF), thionyl chloride $\left(\mathrm{SOCl}_{2}\right)$, triethylamine $\left(\mathrm{Et}_{3} \mathrm{~N}\right)$, toluene $\left(\mathrm{C}_{7} \mathrm{H}_{8}\right)$, ethanol $\left(\mathrm{C}_{2} \mathrm{H}_{5} \mathrm{OH}\right)$, 3-aminopropyl triethoxysilane (APTES) and trichloroacetic acid $\left(\mathrm{C}_{2} \mathrm{HCl}_{3} \mathrm{O}_{2}\right)$ were used as received from Sinopharm Chemical Reagent Co., Ltd.

\subsection{Synthesis of HNTs-decorated rGO (HNTs- $d$-rGO)}

The synthetic routes of acylated $\mathrm{rGO}$ (rGO-COCl), ammoniated HNTs (HNTs-NH ${ }_{2}$ ), HNTs- $d$-rGO are as shown in Figure 1. HNTs- $\mathrm{NH}_{2}$ was synthesized according to the literature [22, 23]. Firstly, $20 \mathrm{~mL}$ APTES was dissolved in $250 \mathrm{~mL}$ of toluene, and then $6 \mathrm{~g}$ of dried HNTs was added into the above solution. The suspension was dispersed ultrasonically for $30 \mathrm{~min}$ at room temperature followed by stirring at $75^{\circ} \mathrm{C}$ for $20 \mathrm{~h}$. The resulting powder was filtered and washed with toluene to remove the excess APTES, and then dried at $120^{\circ} \mathrm{C}$ under vacuum. Graphite oxide $(600 \mathrm{mg})$ prepared using modified Hummers method [24, 25], was dispersed in $\mathrm{SOCl}_{2}$ $(120 \mathrm{~mL})$ with addition of a few drops of DMF $(3 \mathrm{~mL})$, and the mixture was refluxed at $70^{\circ} \mathrm{C}$ for $24 \mathrm{~h}$. After evaporation of the excess $\mathrm{SOCl}_{2}$ under reduced pressure, the $\mathrm{rGO}-\mathrm{COCl}$ product was added into an intensely stirred solution of $200 \mathrm{~mL}$ DMF and $10 \mathrm{~mL}$ triethylamine $\left(\mathrm{Et}_{3} \mathrm{~N}\right)$ at $120^{\circ} \mathrm{C}$ for $36 \mathrm{~h}$. After cooling down to the room temperature, the products of HNTs- $d$-rGO were filtered through a PTFE membrane $(0.22 \mu \mathrm{m})$, and washed with DMF and ethanol for three cycles respectively. Finally, the resulted $\mathrm{HNTs}-d$-rGO was dried in vacuum at $120^{\circ} \mathrm{C}$ for $12 \mathrm{~h}$.

\subsection{Preparation of the PA6/ HNTs- $d$-rGO nanocomposites}

All samples were dried in vacuum at $120^{\circ} \mathrm{C}$ for $24 \mathrm{~h}$ before use. Then the required proportion of HNTs$d$-rGO were mixed and melt blended with PA6 in ThermoHaake Rheomix with the temperature range of $225-250^{\circ} \mathrm{C}$ at screw speed of $80 \mathrm{rpm}$. For comparison, PA6/HNTs, PA6/GO and PA6/mixture of HNTs and GO (HNTs- $m$-GO) were similarly prepared as mentioned for PA6/HNTs- $d$-rGO with equivalent filler content.

\subsection{Characterization}

Fourier-transform infrared (FTIR) spectra were recorded on a Nicolet 8700 FTIR spectrometer. The sample was mixed with potassium bromide $(\mathrm{KBr})$ powder and pressed into pellets. Transmission electron microscopy (TEM) analysis was conducted using a JEOL JEM-2100 instrument with an acceleration voltage of $100 \mathrm{kV}$. The HNTs, GO and HNTs$d$-rGO were dropped on a copper grid for TEM characterization. X-ray photoelectron spectroscopy (XPS) was performed with a VG ESCALB MK-II electron spectrometer. The excitation source was an

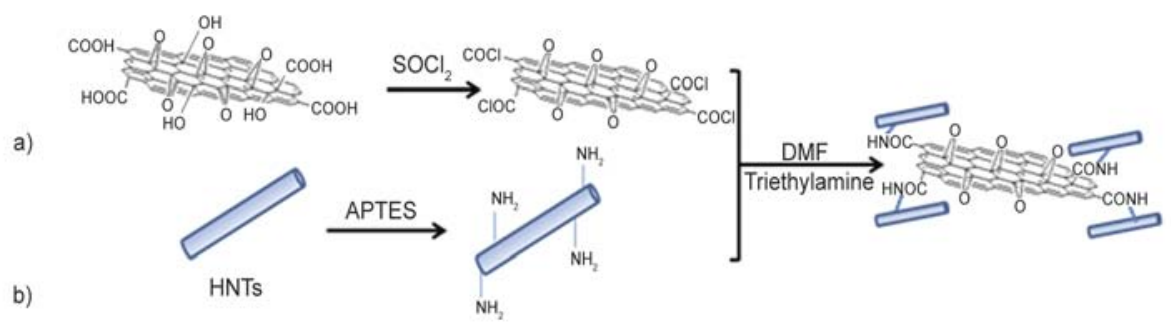

Figure 1. Schematic illustration of the synthetic route of HNTs- $d$-rGO 
$\mathrm{Al} \mathrm{K} \alpha$ line at $1253.6 \mathrm{eV}$. Scanning electron microscopic (SEM) images of the fracture surface of composites were collected using a Hitachi S-4800 fieldemission SEM instrument (operated at $10 \mathrm{kV}$ ). The PA6 nanocomposite pellets were oven-dried and molded into standard $100 \times 100 \times 3 \mathrm{~mm}^{3}$ samples using a Dehong SZ-5-C injection molding machine with the barrel and mold temperatures maintained at 270 and $50^{\circ} \mathrm{C}$, respectively. Then cone calorimeter tests (ISO 5660) were performed on samples placed horizontally with a flux of $35 \mathrm{~kW} / \mathrm{m}^{2}$ to evaluate the flammability of the composites.

\section{Results and discussion \\ 3.1. Characterization of HNTs- $d$-rGO nanostructure}

FTIR spectra of HNTs, HNT-NH ${ }_{2}$, GO and HNTs$d$-rGO are presented in Figure 2. Compared with the pristine HNTs (Figure 2 curve a), HNT-NH $\mathrm{NH}_{2}$ exhibit some new FTIR peaks (Figure 2 curve b), such as the stretching vibration band of $\mathrm{CH}_{2}$ around $2930 \mathrm{~cm}^{-1}$, the deformation vibration $\mathrm{CH}_{2}$ at $1492 \mathrm{~cm}^{-1}$, and the deformation vibration $\mathrm{NH}_{2}$ at $1560 \mathrm{~cm}^{-1}$. All of these show that the successful grafting of amino groups to the surface of HNT. As shown in Figure 2 curve c, the absorption bands of GO at 1719,1618 and $1055 \mathrm{~cm}^{-1}$ are ascribed to $\mathrm{C}=\mathrm{O}, \mathrm{C}=\mathrm{C}$ and $\mathrm{C}-\mathrm{O}[26,27]$, respectively. After the functionalization of $\mathrm{GO}$ by $\mathrm{HNT}-\mathrm{NH}_{2}$, there are some new absorption peaks in the spectrum of HNTs- $d$-rGO. The strong absorption band at around $1090 \mathrm{~cm}^{-1}$ is attributed to the $\mathrm{Si}-\mathrm{O}-\mathrm{Si}$ group in APTES-modified sample HNT- $\mathrm{NH}_{2}$, and the peak at $1568 \mathrm{~cm}^{-1}$ is corresponding to the bending vibrations of the amide groups, which together with the

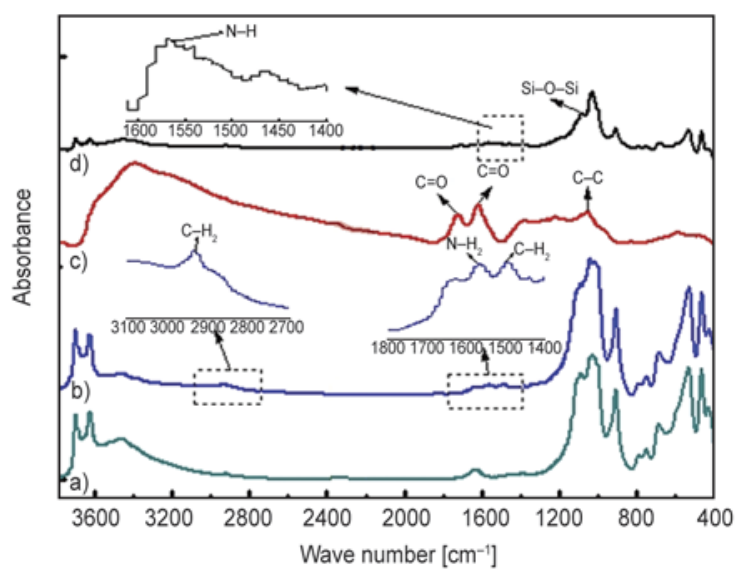

Figure 2. FTIR spectra of (a) pristine HNTs, (b) HNT-NH ${ }_{2}$, (c) GO and (d) HNTs- $d$-rGO significantly reduced intensity of hydroxyl stretching at $3400 \mathrm{~cm}^{-1}$ implying fewer oxygen-containing groups in the reduced $\mathrm{GO}$ are certifications of the successful decoration of HNT- $\mathrm{NH}_{2}$ onto the surface of rGO.

The XPS spectra of pristine HNTs, HNT-NH $\mathrm{N}_{2}$, GO and HNTs- $d$-rGO, and higher resolution $\mathrm{C} 1 \mathrm{~s}$ spectra of GO, HNTs- $d$-rGO and N1s spectra of HNTs$d$-rGO are shown in Figure 3. As can be observed, HNT-NH ${ }_{2}$ displayed a strong photoemission at a binding energy (BE) of $401.9 \mathrm{eV}$ belonging to N1s. The concentration of nitrogen increased up to $4.33 \%$ and the $\mathrm{BE}$ of $401.9 \mathrm{eV}$ assigned to $\mathrm{C}-\mathrm{N}-\mathrm{H}$, which implied the successful ammonification of HNTs. The main Si2p peak at $103 \mathrm{eV}$ is attributed to $\mathrm{Si}-\mathrm{O}-\mathrm{Si} / \mathrm{Si}-\mathrm{O}-\mathrm{Al}[23,28]$. The C1s XPS of GO (Figure 3b) shows a degree of oxidation with four peaks appearing at 284.8, 285.6, 286.8 and $288.4 \mathrm{eV}$, which are ascribed to the non-oxygenated ring $\mathrm{C}$, the $\mathrm{C}$ bonded to $\mathrm{C}$ atoms in defective structures, the $\mathrm{C}$ in $\mathrm{C}-\mathrm{O}$ bonds and the carbonyl $\mathrm{C}$, respectively [29]. The C1s XPS spectrum of HNTs- $d$-rGO (Figure $3 \mathrm{c}$ ) also exhibits these same oxygen functionalities, but the peak intensities of oxygenated $\mathrm{C}$ are much smaller than those in GO, indicating the partial reduction of GO. In the N1s spectra of HNTs- $d$ rGO (Figure 3d), The detection of a N1s peak at a binding energy of $400.6 \mathrm{eV}$, which is ascribed to $\mathrm{C}-\mathrm{N}-\mathrm{C}$, is a key indication of the successful covalent functionlization of rGO by HNTs.

On the basis of FTIR and XPS results, TEM was employed to obtain the morphological information of products we have synthesized. As shown in Figure 4, compared with pristine HNTs (Figure 4a), HNT-NH ${ }_{2}$, which was prepared by amino-functionalized HNT using APTES, not only did the diameter of the HNT increase to some degree, but also their surfaces (including outer surface and inner surface) were much rougher relative to HNT, and a thin polymeric layer could be observed (Figure $4 \mathrm{~b}$ ). The layers of GO (Figure 4c) are clean and several hundred nanometers large, while HNTs- $d$-rGO (Figure 4d) have many nanotubes attached. The rGO in HNTs- $d$-rGO was so thin that some of the edges are nearly invisible (Figure 4d). Moreover, the ordered graphite lattices are clearly visible and the disordered regions are also found in Figure 4e, indicating that the GO sheets were partially reduced and restored to ordered crystal structure. The inset selected area electron diffraction pattern (SAED), 

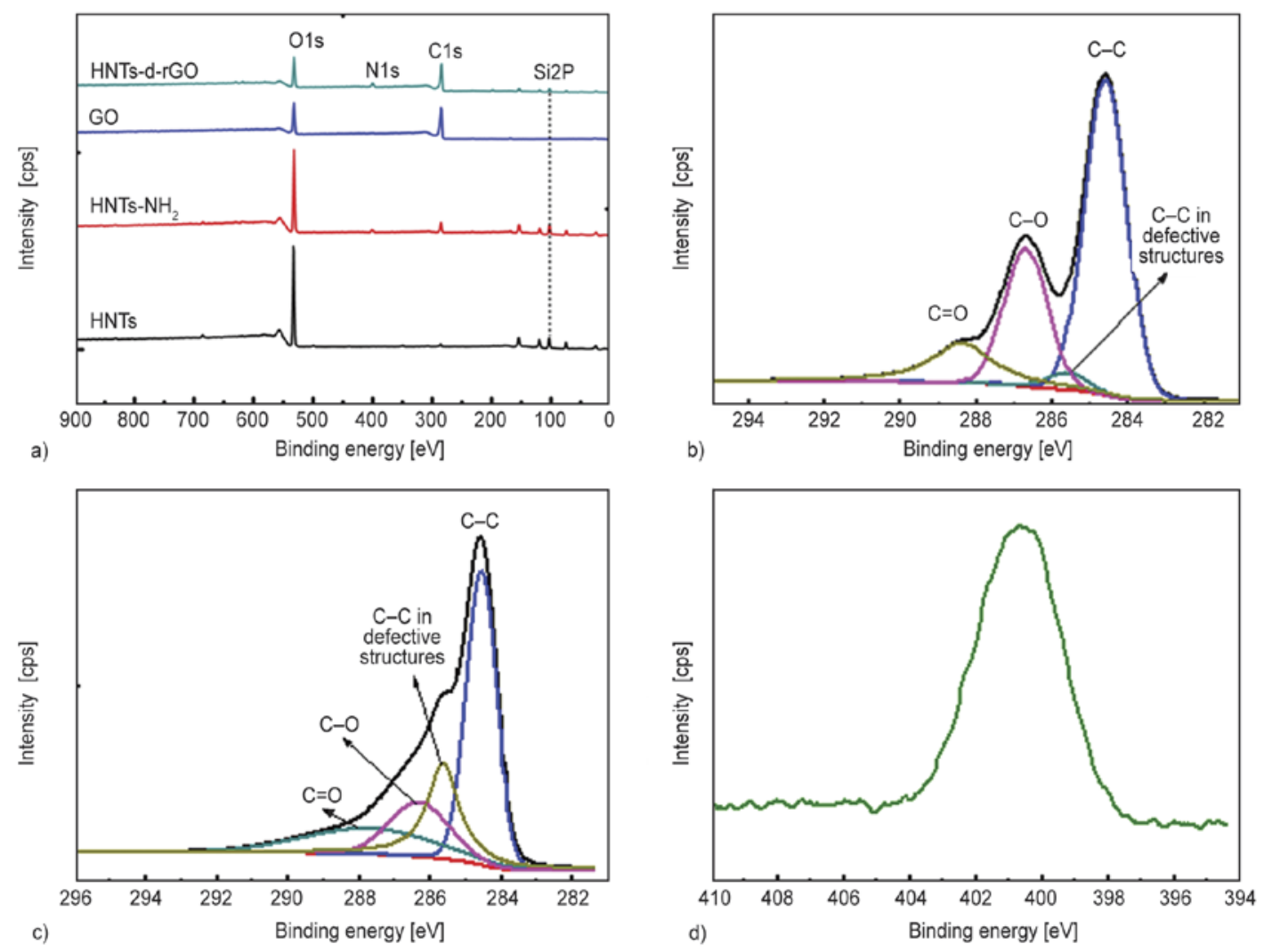

Figure 3. XPS spectra of (a) pristine HNTs, HNT-NH $\mathrm{H}_{2}$, GO and HNTs- $d$-rGO, and higher resolution C1s spectra of (b) GO, (c) HNTs- $d$-rGO and (d) N1s spectra of HNTs- $d$-rGO

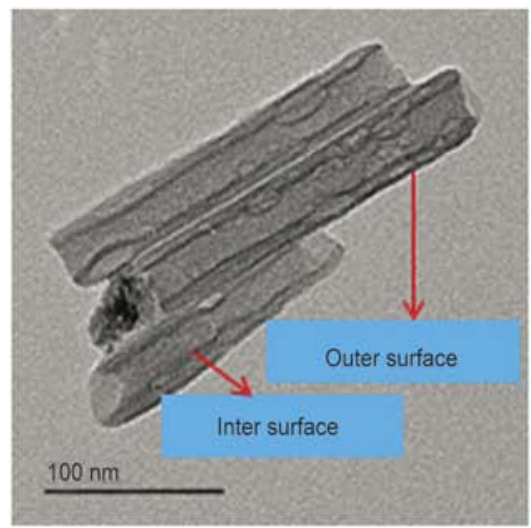

a)

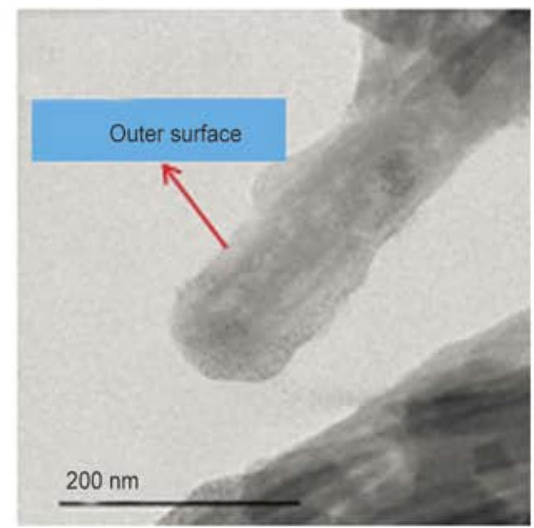

b)

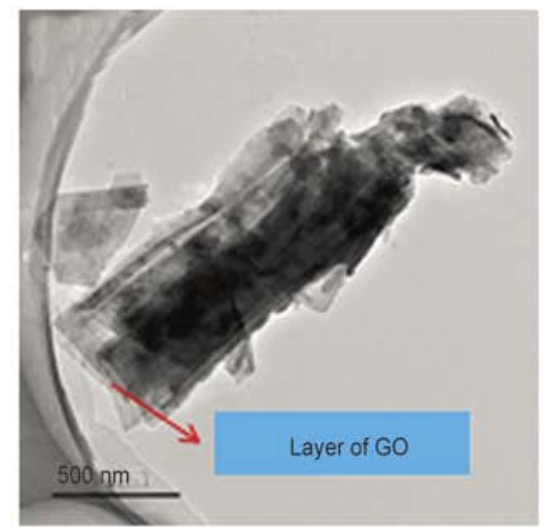

c)

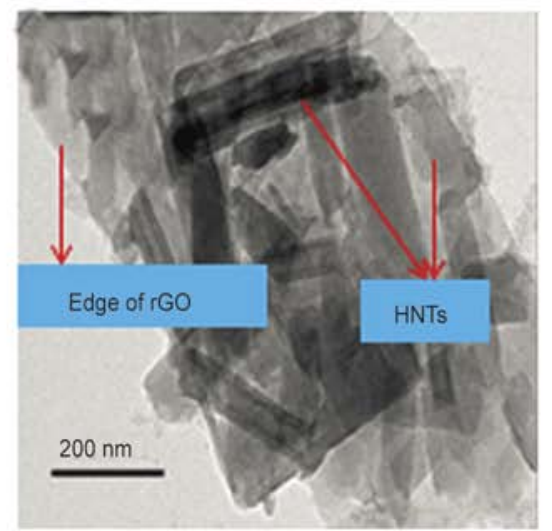

d)

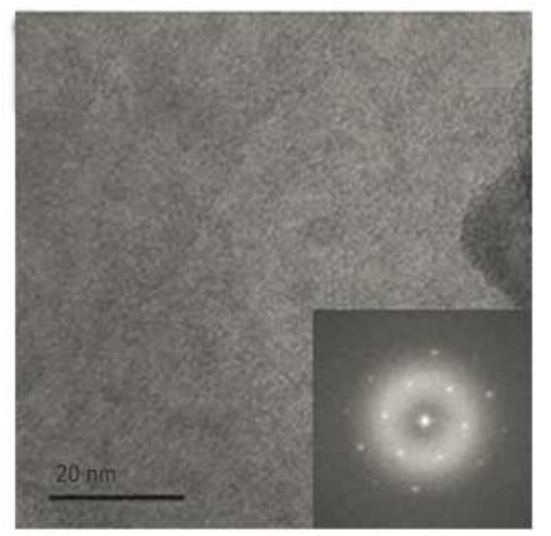

e)

Figure 4. TEM images of (a) pristine HNTs, (b) HNT-NH ${ }_{2}$, (c) GO, (d) HNTs- $d$-rGO, (e) HRTEM of the rGO in HNTs- $d$ rGO (inset is the SAED pattern) 
shows both diffraction rings and dots, which is consistent with high resolution transmission electron microscopy (HRTEM) results.

\subsection{Dispersion of HNTs- $d$-rGO in the composites}

The dispersion of fillers in PA6 is an important factor to affect the various properties of composites. It is necessary to obtain the morphological information of fillers in the matrix. In this study, SEM was used to examine the dispersion of HNTs, GO, HNTs$d$-rGO and HNTs- $m$-rGO in PA6. In order to show the dispersion state more clearly, the fractured surfaces of PA6 composites made by cryo-fractured in liquid nitrogen were etched with $\mathrm{C}_{2} \mathrm{HCl}_{3} \mathrm{O}_{2}$ $(30 \mathrm{wt} \%) / \mathrm{C}_{2} \mathrm{H}_{5} \mathrm{OH}$ solution at $60^{\circ} \mathrm{C}$ for $1 \mathrm{~h}$. It is evident that HNTs are homogeneously dispersed in the PA6/HNTs composites (Figure 5a). As shown in Figure 5b, many GO sheets could be observed on the fractured surface and they are uniformly dis-

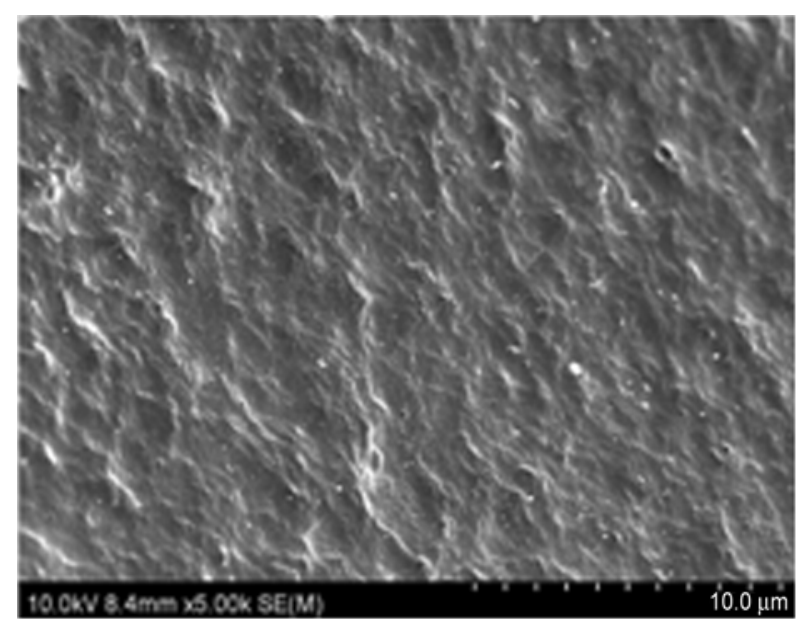

a)

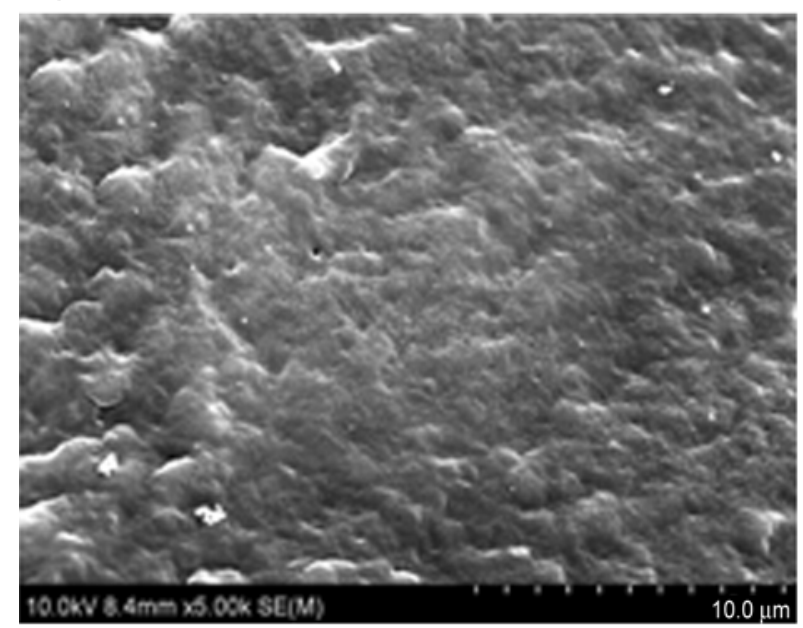

c) persed in the PA6/GO composites without agglomeration. Figure $5 \mathrm{c}$ presents a good dispersion of HNTs- $d$-rGO in PA6 matrix and less isolated HNTs and their aggregates compared with those in PA6/ HNTs- $m$-rGO composite (as shown in Figure 5d), indicating a strong interaction between the $\mathrm{rGO}$ sheets and the HNTs, which may be good for the flame retardant property of PA6 composite.

\subsection{Flame-retardant properties of the PA6 composites}

The cone calorimeter test results for PA6 and the corresponding composites filled with 1 wt.\% of HNTs, GO and HNTs- $d$-rGO prepared by melt blending are presented in Figure 6 and Table 1. From Figure 6a, it can be observed that pure PA6 burns rapidly after ignition and the peak heat release rate (PHRR) value is $663 \mathrm{~kW} / \mathrm{m}^{2}$. As expected, incorporating HNTs and GO into PA6 makes the PHRR decrease to 634 and $617 \mathrm{~kW} / \mathrm{m}^{2}$ respectively. Moreover, the PHRR

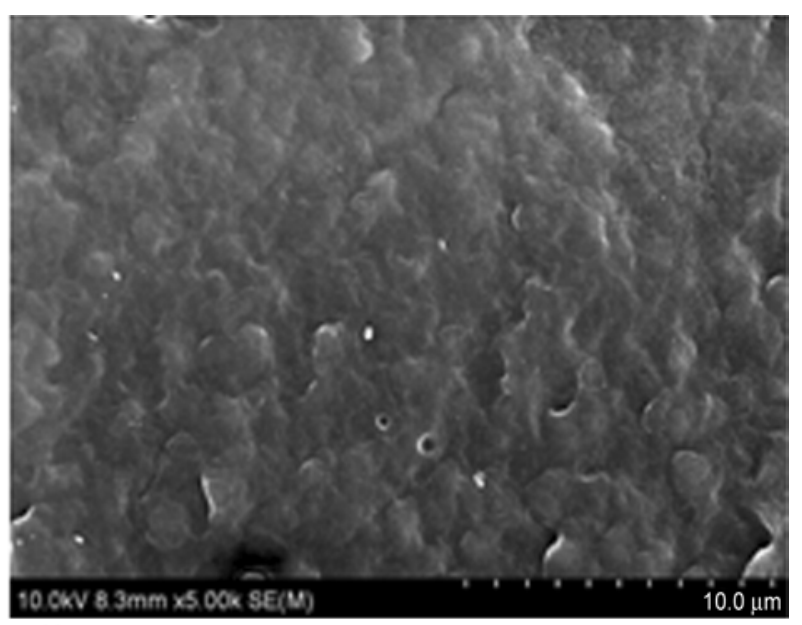

b)

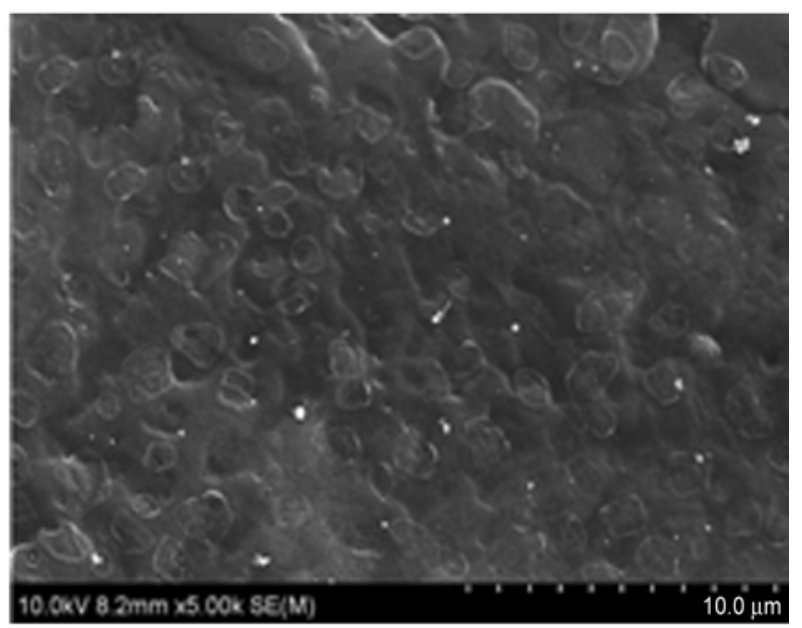

d)

Figure 5. SEM images obtained from fracture surface of $1.0 \mathrm{wt} \%$ (a) pristine HNTs, (b) GO, (c) HNTs- $d$-rGO, (d) HNTs$m$-GO in PA6 matrix etched with $30 \mathrm{wt} \%$ trichloroacetic acid/ethanol solution at $60^{\circ} \mathrm{C}$ for $1 \mathrm{~h}$ 


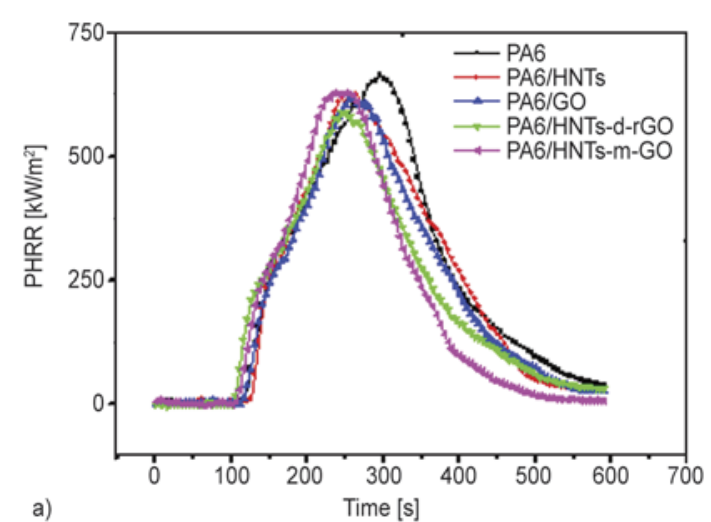

Figure 6. Heat release rate (HRR) (a), total heat release (THR) (b) versus time curves of polyamide 6

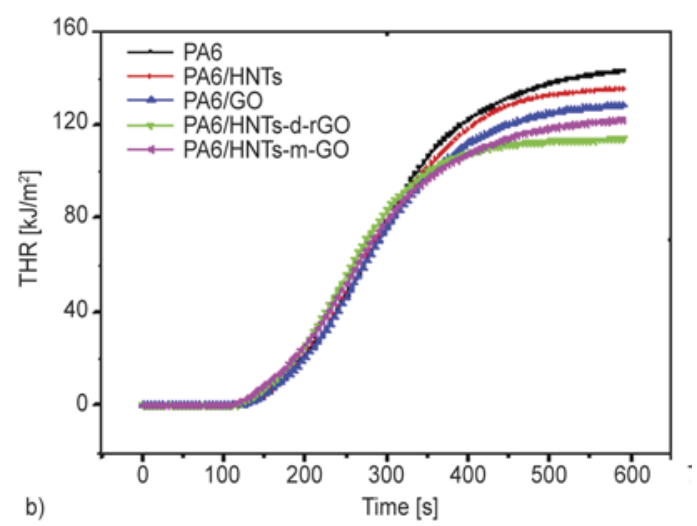

Table 1. Cone test results for PA6 and PA6 composites

\begin{tabular}{|l|c|c|c|c|}
\hline \multicolumn{1}{|c|}{ Samples } & $\begin{array}{c}\text { PHRR } \\
{\left[\mathbf{k W} / \mathbf{m}^{\mathbf{2}}\right]}\end{array}$ & $\begin{array}{c}\text { THR } \\
{\left[\mathbf{M J} / \mathbf{m}^{\mathbf{2}}\right]}\end{array}$ & $\begin{array}{c}\text { TTI } \\
{[\mathbf{s}]}\end{array}$ & $\begin{array}{c}\mathbf{T S P} \\
{\left[\mathbf{m}^{\mathbf{2}} / \mathbf{m}^{\mathbf{2}}\right]}\end{array}$ \\
\hline PA6 & 663 & 142.6 & 110 & 4.26 \\
\hline PA6/HNTs & 634 & 135.0 & 121 & 4.35 \\
\hline PA6/GO & 617 & 128.2 & 99 & 4.98 \\
\hline PA6/HNTs- $d$-rGO & 588 & 113.6 & 107 & 4.72 \\
\hline PA6/HNTs- $m$-GO & 628 & 122.0 & 104 & 4.70 \\
\hline
\end{tabular}

of PA6/HNTs- $d$-rGO composite exhibits a further reduction compared to both PA6/HNTs and PA6/GO. To clarify whether the grafting effect or the effect of a simple combination of HNTs and CNTs was responsible for this result, we conducted a contrast experiment by physically mixing HNTs and GO in PA6. The PHRR for this composite was higher than HNTs- $d$-rGO at the same loading level, indicating the grafting was good for the fire retardancy. Therefore, the best fire retardant properties of PA6/HNTs- $d$-rGO could be attributed to two aspects: firstly, the reduction of GO converts GO into a more stable form, reduced GO (rGO); Secondly, HNTs can create a stable silica layer on the char surface of PA6, which reinforces the barrier effect of graphene. From Figure 6b, The total heat release (THR) curves show a similar trend as heat release rate (HRR), and THR values of PA6/ HNTs, PA6/GO, PA6/HNTs- $d$-rGO are reduced from $142.6 \mathrm{MJ} / \mathrm{m}^{2}$ (pure PA6) to $135.0,128.2$ and $113.6 \mathrm{MJ} / \mathrm{m}^{2}$, respectively. While the PA6/HNTs$m$-GO exhibits the higher THR value than PA6/ HNTs- $d$-rGO, which proves the above mechanism for the best fire retardant properties of PA6/HNTs$d$-rGO again. From Table 1, it can be found that incorporation of the nanofillers has a slight influence on the time-to-ignition (TTI) of the composites, owing to their small loadings. With regard to the total smoke production (TSP), all the samples containing nanofillers show higher TSP compared to pure PA6. This phenomenon is probably due to the incomplete combustion of PA6.

\section{Conclusions}

In summary, we have firstly reported the covalently bonded HNT- $d$-rGO nanostructures, which successfully improved the flame-retardant properties as the fillers in PA6 from the PHRR and THR results. The nanostructures of HNT- $d$-rGO were determined by FTIR, XPS, and TEM studies. A morphological study revealed that HNTs- $d$-rGO was dispersed well in PA6. Our results indicate that the improvement in flame-retardancy of HNT- $d$ rGO hybrid composite may due to the barrier effect of its network. The HNT- $d$-rGO hybrid composites open up a new strategy to be applied towards a wide range of polymers materials as an effective fillers to improve the fire safety of polar polymers.

\section{Acknowledgements}

This study is supported by The National Science Fund for Distinguished Young Scholars (50925312), Program of Changjiang Scholars and Innovative Research Team in University (T2011079, IRT1221) and Research Program of Shanghai Science and Technology Commission (12nm

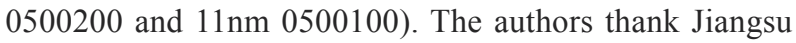
Haiyang Chemical Fiber Co., Ltd. for kindly providing the PA6 samples used in this study.

\section{References}

[1] Kim G-M., Michler G. H., Ania F., Balta Calleja F. J.: Temperature dependence of polymorphism in electrospun nanofibres of PA6 and PA6/clay nanocomposite. Polymer, 48, 4814-4823 (2007).

DOI: $10.1016 /$ j.polymer.2007.05.082 
[2] Ryu Y. J., Kim H. Y., Lee K. H., Park H. C., Lee D. R.: Transport properties of electrospun nylon 6 nonwoven mats. European Polymer Journal, 39, 1883-1889 (2003). DOI: 10.1016/S0014-3057(03)00096-X

[3] Cai Y., Huang F., Wei Q., Song L., Hu Y., Ye Y., Xu Y., Gao W.: Structure, morphology, thermal stability and carbonization mechanism studies of electrospun PA6/ Fe-OMT nanocomposite fibers. Polymer Degradation and Stability, 93, 2180-2185 (2008). DOI: $10.1016 /$ j.polymdegradstab.2008.08.003

[4] Abacha N., Kubouchi M., Sakai T.: Diffusion behavior of water in polyamide 6 organoclay nanocomposites. Express Polymer Letters, 3, 245-255 (2009).

DOI: $10.3144 /$ expresspolymlett.2009.31

[5] Zang J., Ryu S., Pugno N., Wang Q., Tu Q., Buehler M. J., Zhao X.: Multifunctionality and control of the crumpling and unfolding of large-area graphene. Nature Materials, 12, 321-325 (2013).

DOI: 10.1038/NMAT3542

[6] Fardindoost S., Mohammadi S., Iraji zad A., Sarvari R., Shariat Panahi S. P., Jokar E.: Electromechanical resonator based on electrostatically actuated graphenedoped PVP nanofibers. Nanotechnology, 24, 135201/1135201/6 (2013).

DOI: $10.1088 / 0957-4484 / 24 / 13 / 135201$

[7] Majumdar A., Kim J., Vuckovic J., Wang F.: Electrical control of silicon photonic crystal cavity by graphene. Nano Letters, 13, 515-518 (2013).

DOI: $10.1021 / \mathrm{nl} 3039212$

[8] Prakash A., Misra S. K., Bahadur D.: The role of reduced graphene oxide capping on defect induced ferromagnetism of $\mathrm{ZnO}$ nanorods. Nanotechnology, 24, 095705/1-095705/10 (2013).

DOI: 10.1088/0957-4484/24/9/095705

[9] Veca L. M., Meziani M. J., Wang W., Wang X., Lu F., Zhang P., Lin Y., Fee R., Connell J. W., Sun Y-P.: Carbon nanosheets for polymeric nanocomposites with high thermal conductivity. Advanced Materials, 21, 2088-2092 (2009).

DOI: $10.1002 /$ adma.200802317

[10] Balandin A. A., Ghosh S., Bao W. Z., Calizo I., Teweldebrhan D., Miao F., Lau C. N.: Superior thermal conductivity of single-layer graphene. Nano Letters, 8 , 902-907 (2008).

DOI: $10.1021 / \mathrm{n} 10731872$

[11] Huang G., Gao J., Wang X., Liang H., Ge C.: How can graphene reduce the flammability of polymer nanocomposites? Materials Letters, 66, 187-189 (2012).

DOI: $10.1016 /$ j.matlet.2011.08.063

[12] Bao C., Song L., Wilkie C. A., Yuan B., Guo Y., Hu Y., Gong X.: Graphite oxide, graphene, and metal-loaded graphene for fire safety applications of polystyrene. Journal of Materials Chemistry, 22, 16399-16406 (2012).

DOI: $10.1039 / \mathrm{c} 2 \mathrm{jm} 32500 \mathrm{~d}$
[13] Song P., Liu L., Fu S., Yu Y., Jin C., Wu Q., Zhang Y., Li Q.: Striking multiple synergies created by combining reduced graphene oxides and carbon nanotubes for polymer nanocomposites. Nanotechnology, 24, 125704/1-125704/8 (2013).

DOI: 10.1088/0957-4484/24/12/125704

[14] Pack S., Kashiwagi T., Cao C. H., Korach C. S., Lewin M., Rafailovich M. H.: Role of surface interactions in the synergizing polymer/clay flame retardant properties. Macromolecules, 43, 5338-5351 (2010). DOI: $10.1021 / \mathrm{ma100669g}$

[15] Dasari A., Yu Z-Z., Mai Y-W., Liu S.: Flame retardancy of highly filled polyamide 6/clay nanocomposites. Nanotechnology, 18, 445602/1-445602/10 (2007). DOI: $10.1088 / 0957-4484 / 18 / 44 / 445602$

[16] Handge U. A., Hedicke-Höchstötter K., Altstädt V.: Composites of polyamide 6 and silicate nanotubes of the mineral halloysite: Influence of molecular weight on thermal, mechanical and rheological properties. Polymer, 51, 2690-2699 (2010).

DOI: $10.1016 /$ j.polymer.2010.04.041

[17] Liu M., Guo B., Zou Q., Du M., Jia D.: Interactions between halloysite nanotubes and 2,5-bis(2-benzoxazolyl) thiophene and their effects on reinforcement of polypropylene/halloysite nanocomposites. Nanotechnology, 19, 205709/1-205709/10 (2008).

DOI: $10.1088 / 0957-4484 / 19 / 20 / 205709$

[18] Liu C., Luo Y. F., Jia X. X., Zhong B. C., Li S. Q., Guo B. C., Jia D. M.: Enhancement of mechanical properties of poly(vinyl chloride) with polymethyl methacrylate-grafted halloysite nanotube. Express Polymer Letters, 5, 591-603 (2011).

DOI: $10.3144 /$ expresspolymlett.2011.58

[19] Liu M. X., Guo B. C., Du M. L., Chen F., Jia D. M.: Halloysite nanotubes as a novel $\beta$-nucleating agent for isotactic polypropylene. Polymer, 50, 3022-3030 (2009).

DOI: $10.1016 /$ j.polymer.2009.04.052

[20] Guo B., Zou Q., Lei Y., Du M., Liu M., Jia D.: Crystallization behavior of polyamide 6/halloysite nanotubes nanocomposites. Thermochimica Acta, 484, 48-56 (2009).

DOI: $\underline{10.1016 / \mathrm{j} . t c a .2008 .12 .003}$

[21] Marney D. C. O., Russell L. J., Wu D. Y., Nguyen T., Cramm D., Rigopoulos N., Wright N., Greaves M.: The suitability of halloysite nanotubes as a fire retardant for nylon 6. Polymer Degradation and Stability, 93, 1971-1978 (2008).

DOI: 10.1016/j.polymdegradstab.2008.06.018

[22] Yuan P., Southon P. D., Liu Z., Green M. E. R., Hook J. M., Antill S. J., Kepert C. J.: Functionalization of halloysite clay nanotubes by grafting with $\gamma$-aminopropyltriethoxysilane. Journal of Physical Chemistry C, 112, 15742-15751 (2008).

DOI: $10.1021 / \mathrm{jp} 805657 \mathrm{t}$ 
[23] Yah W. O., Takahara A., Lvov Y. M.: Selective modification of halloysite lumen with octadecylphosphonic acid: New inorganic tubular micelle. Journal of the American Chemical Society, 134, 1853-1859 (2012). DOI: $10.1021 /$ ja210258y

[24] Hummers W. S., Offeman R. E.: Preparation of graphitic oxide. Journal of the American Chemical Society, 80, 1339-1339 (1958).

DOI: $10.1021 / \mathrm{ja} 01539 \mathrm{a} 017$

[25] Petit C., Bandosz T. J.: MOF-graphite oxide composites: Combining the uniqueness of graphene layers and metal-organic frameworks. Advanced Materials, 21, 4753-4757 (2009).

DOI: 10.1002/adma.200901581

[26] Bagri A., Mattevi C., Acik M., Chabal Y. J., Chhowalla M., Shenoy V. B.: Structural evolution during the reduction of chemically derived graphene oxide. Nature Chemistry, 2, 581-587 (2010).

DOI: 10.1038 /NCHEM.686
[27] Ou J., Wang J., Liu S., Mu B., Ren J., Wang H., Yang S.: Tribology study of reduced graphene oxide sheets on silicon substrate synthesized via covalent assembly. Langmuir, 26, 15830-15836 (2010). DOI: $10.1021 / 1 \mathrm{a} 102862 \mathrm{~d}$

[28] Wittberg T. N., Wang P. S.: XPS study of the dehydration of clay and kaolin powders. Surface and Interface Analysis, 27, 936-940 (1999).

DOI: 10.1002/(SICI)1096-9918(199910)27:10<936::

$$
\text { AID-SIA656>3.0.CO;2-N }
$$

[29] Stankovich S., Dikin D. A., Piner R. D., Kohlhaas K. A., Kleinhammes A., Jia Y., Wu Y., Nguyen S. T., Ruoff R. S.: Synthesis of graphene-based nanosheets via chemical reduction of exfoliated graphite oxide. Carbon, 45, 1558-1565 (2007).

DOI: 10.1016/j.carbon.2007.02.034 